\title{
DETERMINING THE COLOR OF THE TEETH BY USING DENTAL IMAGES WITH ISOLATION OF GINGIVAL COLOR
}

\author{
Radostina Vasileva \\ Dental Material Science and Propedeutics of Prosthetic Dental Medicine, \\ Faculty of Dental Medicine, Medical University of Varna
}

\begin{abstract}
INTRODUCTION: As a difficult and subjective process, when we determine the color of the teeth, we often use additional methods. One of the methods for color definition when communicating with a technical dental laboratory is the use of photography.

AIM: The aim of this study was to compare the color definition using a photo in terms of isolating the gingival color and without isolation of the gingival color. The presence of a background and distracting colors has a serious impact on color determination. Placed under different conditions, the colors are interpreted in a different way. For the needs of dental medicine the accurate color determination is of vital importance. This is particularly true in case of absence of a direct communication between a laboratory and a dental office. The actual color definition is done by a photo.
\end{abstract}

MATERIALS AND METHODS: For the purpose of the study, a Shofu Eye Special 2 camera was used, specially created for the dental photography needs. The device had a built-in menu option to isolate the gingiva. The survey methodology included visual color determination of 2 images with a placed coloring (shade master) of Vita.

RESULTS: The results of this study, show a unique advantage when defining the color with gingival color isolation. This happens especially visibly in a closer contact of the color standard with the masked background.

CONCLUSION: The color definition option by masking the gingival background is highly effective and convenient to determine the color of teeth for the needs of dentistry.

Keywords: dental images, dental photography, color, isolation of color

Address for correspondence:

Radostina Vasileva

Faculty of Dental Medicine

Medical University of Varna

84 Tzar Osvoboditel Blvd

9000 Varna

e-mail: radost11dent@abv.bg

Received: September 3, 2018

Accepted: December 21, 2018

\section{INTRODUCTION}

As a difficult and subjective process, when we determine the color of the teeth, often use additional methods. The use of photography for dentistry needs is an established standard for a good practice (1-5). It is also used successfully as an auxiliary method for color definition (1-3, 6-10). Color determination in dental medicine has always been difficult and de- 
pending on many factors. One of the problems is the impossibility of direct color determination by a dental technician. Using a digital image is one of the decisions. The color definition itself is influenced by factors such as background, source light, distance and more $(6,10)$.

The background as a condition has an important meaning in color determination for digital images. The separation of the distracting color from tooth color, is a method of prime importance. Multiple authors offer color definition protocols by the help of dental photography. The applied methodology as an option with the digital apparatus Shofu Eye Special C 2, which is a convenient and appropriate appliance for dental medicine doctors.

\section{AIM}

The aim of this article is to determine the importance of the distracting gingival colors as an important condition for the determination of the exact tooth color.

\section{MATERIALS AND METHODS}

For the purpose of the study, a Shofu Eye Special 2 camera was used, specially created for the dental photography needs. The device had a built-in menu option to isolate the gingiva. This option allowed all the colors of the image, the colors of the teeth in the teeth rows, to convert to black and white.

For the purpose of the study, we first shot and determined the color of the dental arch, using DSLR Cannon, Color Checker master camera and CIE LAB digital image analysis, specifying $3 \mathrm{M} 1$ as an accurate color.

The survey methodology included visual color determination of 2 images with a placed coloring (shade master) of Vita 3D Master with an exact color similar to the tooth color - 3M1 and one model with a close color - 3M2, as on one of the pictures the gingival color was not isolated, and on the other image it was isolated (Fig. 1, 2). The photos were captured in JPEG format, without post-editing adjustments. The images were printed on Kodak photographic paper, then handed over to a number of dental technicians and doctors in dental medicine. The analysis of all the results was performed using IBM SPSS Statistics 19 software.

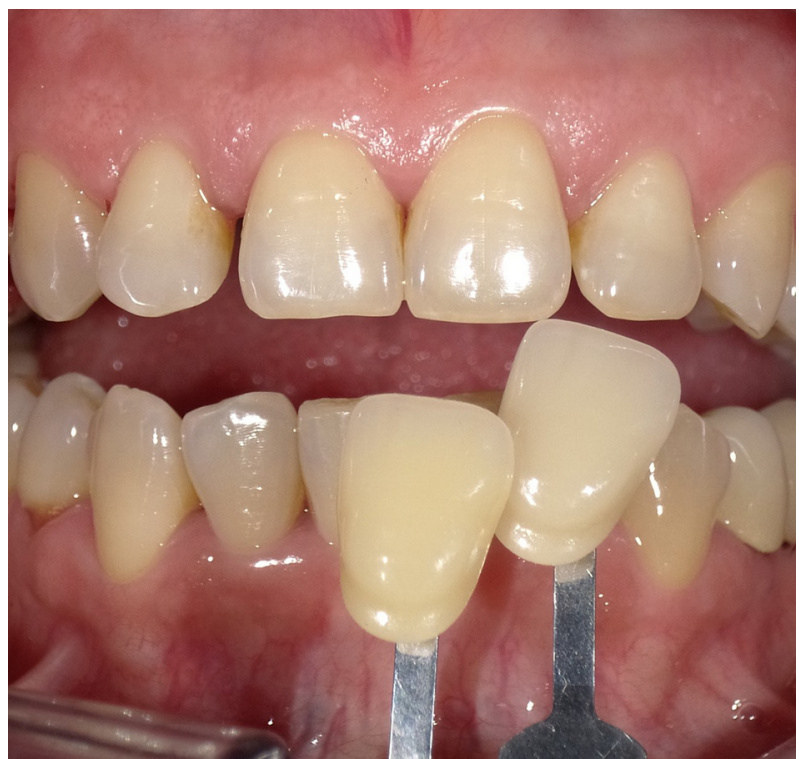

Fig. 1. Image, without isolated gingival color, and with placed shade master Vita 3D Master-3M1 and 3M2

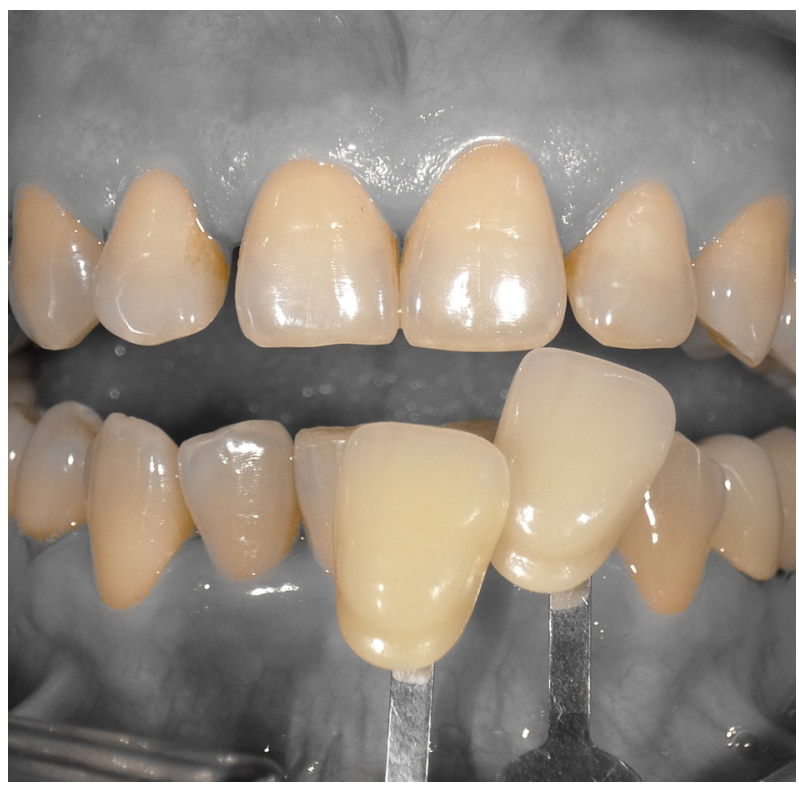

Fig. 2. Image, with isolated gingival color, and with placed shade master Vita 3D Master-3M1 and 3M2

\section{RESULTS AND DISCUSSION}

The obtained results were structured as tables when we presented them to specialists divided into groups (Fig. 3). In the processing of the results, information from correctly filled maps of doctors in dental medicine and dental technicians was included. In total 28 specialists participated in the study, including 18 men and 10 middle-aged women at an average 
age of $48 \pm 28.5$. They included 12 dental technicians and 16 doctors in dental medicine. The so divided two groups that we conditionally named $\mathrm{A}$ and $\mathrm{B}$ determined by visual method the exact color according to them, so first they successively described the picture color without isolating the gingiva and then they filled in the color data using an image with an isolated gingival color. The obtained results of the analysis were described and processed statistically.

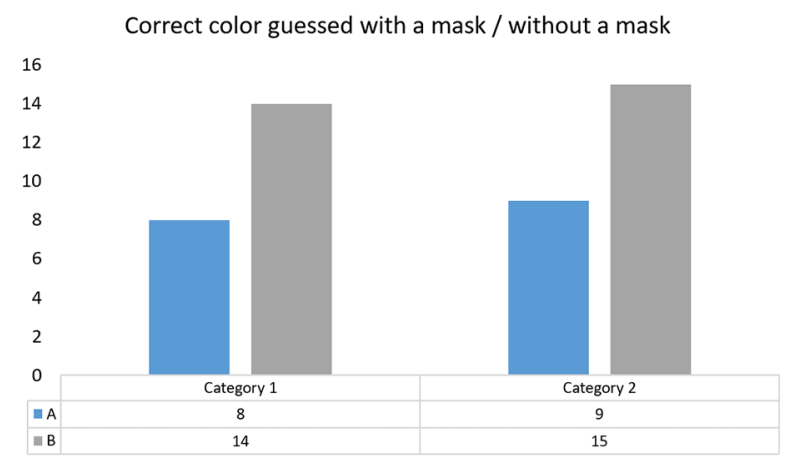

Fig. 3. Result of participants - recognized the correct color

The starting point for the correct color was $3 \mathrm{M} 1$. The ones who guessed the right color in the conditions without mask were 8 people from group A, while under masking conditions the ones who guessed the right color were 14 people. The ones who guessed the correct answer from group $\mathrm{C}$ were 9 people in no-mask conditions and 15 people in mask conditions. The results show a unique advantage when defining the color with gingival color isolation. This happens especially visibly in a closer contact of the color standard with the masked background. The dental doctors determined the color with a clinically insignificant difference compared to the dental technicians.

\section{CONCLUSION}

The color definition option by masking the gingival background is highly effective and convenient to determine the color of teeth for the needs of dentistry. It could be applied both with the help of special dental cameras and via software. The guiding of visual attention to the color of the object and the isolation of the distracting elements are important for a correct color rendering. Despite the obtained results, we think that a further research is needed to finetune the technique for an accurate color rendering and color definition.

\section{REFERENCES}

1. McLaren EA, Schoenbaum T. Digital photography enhances diagnostics, communication, and documentation. Compend Contin Educ Dent. 2011;32(Spec 4):36-8.

2. Ahmad I. Digital dental photography. Part 2: purposes and uses. Br Dent J. 2009 May 9;206(9):459 64. doi: 10.1038/sj.bdj.2009.366.

3. Ahmad I. Digital dental photography. Part 1: an overview. Br Dent J. 2009 Apr 25;206(8):403-7. doi: 10.1038/sj.bdj.2009.306.

4. Mladenovic D, Mladenovic L, Mladenovic S. Importance of digital dental photography in the practice of dentistry. Sci J Faculty Med Niš. 2010;27(2):75-9.

5. Sandler J, Gutierrez RJ, Marray A. Clinical photographs: the gold standard, an update. Prog Orthod. 2012;13(3):296-303. doi: 10.1016/j.pio.2011.12.002.

6. Terry DA, Snow SR, McLaren EA. Contemporary dental photography: selection and application. Compend Contin Educ Dent. 2008;29(8):432-6. 438, 440-2. passim.

7. Christensen GJ. Important clinical uses for digital photography. J Am Dent Assoc. 2005;136(1):77-9.

8. Wander P, Gordon P. Specific applications of dental photography. Br Dent J. 1987;162(10):393-403. doi: 10.1038/sj.bdj.4806143.

9. Ahmad I. Digital dental photography. Part 10: printing, publishing and presentations. Br Dent J. 2009;207(6):261-5. doi: 10.1038/sj.bdj.2009.814.

10. Mahn E. Dental photography. Part II. Protocol for shade taking and communication with the lab. Intern Dentistry - Australasian Edition. 8(2). 